Project 1016387

\title{
Environmental Actinide Mobility: Plutonium and Uranium Interactions with Exopolysaccharides and Siderophores of Aerobic Soil Microbes
}

\author{
Neu, Mary P. \\ Los Alamos National Laboratory \\ Vanderberg, Laura \\ Los Alamos National Laboratory
}

RESULTS TO DATE: Research efforts into understanding the effect of siderophores and capsules on actinide speciation and resultant environmental behavior are ongoing. Investigations on both siderophores have progressed. The redox chemistry of $\mathrm{Pu}(\mathrm{VI})$ and $\mathrm{Pu}(\mathrm{V})$ with DFO under acidic conditions was examined by UV-Vis and NMR spectroscopies. $\mathrm{Pu}(\mathrm{VI})$ was instantly reduced to $\mathrm{Pu}(\mathrm{V})$ by $\mathrm{DFO}$ and $\mathrm{DFO}$ cleavage resulted. Neither DFO nor the cleavage product appeared to coordinate with $\mathrm{Pu}(\mathrm{V})$ in the $\mathrm{pH}$ range examined. Growth of $\mathrm{S}$. pilosus for cellular translocation experiments was optimized and experiments to determine DFO-mediated uranium uptake by S. pilosus are underway. OFS siderophore(s) production has been optimized and product identification is underway. Siderophore-like molecules were positive for the Arnow assay and had UV absorbance bands at $250 \mathrm{~nm}$ and $315 \mathrm{~nm}$, suggesting catecholatestype molecules. Siderophores were produced with C13 - C16 as carbon source in AM-1 salts medium with $1.0 \mathrm{mM}$ iron. Siderophore accumulation was slowest on C13 due to the slower growth of OFS on this substrate. The C16 concentration in the culture influenced siderophore production, with highest levels at 1.0\% and 2.0\%. HPLC confirmed the presence of three aromatic compounds. The absorbance of one of these compounds at $315 \mathrm{~nm}$ had a similar peak structure as that of a 2,3-dihydroxybenzoic acid. 2,3-Dihydroxybenzoic acid siderophores often have an amino acid conjugate. Hydrolysis of semipurified siderophores demonstrated the presence of arginine and an unidentified peak near the retention time of glycine. Further purification, characterization, and metal binding studies are planned. The production of the glutamic acid capsule from Bacillus lichenformis has been optimized and conditions under which it remains stable have been determined. The average molecular weight of isolated polymer was determined by gel permeation chromatography (Mn $\neg 800,000 \mathrm{~g} / \mathrm{mol}$ ). This corresponds to 5,700 glutamate units/polymer. The capsule had zero points of charge from 4.5 to 5.5, depending background electrolyte. Preliminary results indicated a high binding capacity of $\mathrm{U}(\mathrm{VI})$ by the capsule. At $\mathrm{pH} 5.5,70 \%$ of $\mathrm{U}(\mathrm{VI})$ was complexed when the concentrations of $\mathrm{U}(\mathrm{VI})$ and capsule were $10 \mathrm{mM}$ and $0.01 \mathrm{~g} / \mathrm{L}$, respectively, resulting in a binding capacity of $8.02 \mathrm{mmol} \mathrm{U}(\mathrm{VI})$ per gram of capsule. Increasing capsule concentration to $0.1 \mathrm{~g} / \mathrm{L}$ led to complexation of $95 \%$ of the $\mathrm{U}(\mathrm{VI})$. Capsule- $\mathrm{U}(\mathrm{VI})$ complexes were examined by UV-Vis and ATR-FTIR spectroscopies. Ratios of $\mathrm{U}(\mathrm{VI})$ to capsule were normalized to moles of $\mathrm{U}(\mathrm{VI})$ to moles of the glutamate unit. For ratios ranging from 1:1 to 1:5 (U:glutamate subunit) and $\mathrm{pH} 2.5$ the $\mathrm{U}(\mathrm{VI}) /$ capsule flocculated 
and formed white precipitates. Spectra of the filtrates had lower diagnostic absorbance compared to that for pure $\mathrm{U}(\mathrm{VI})$ solution, suggesting that $\mathrm{U}(\mathrm{VI})$ precipitated with the capsule. When the ratio was increased to 1:10, no precipitate was observed and the spectrum changed from that of pure uranyl to that for a soluble uranyl-capsule complex. Under expected environmental conditions (e.g., low polymer concentration, $\mathrm{pH} \sim 5.5$, and low ionic strength) PGA exists in a helical structure. At this $\mathrm{pH}$, trace toxic metals will be strongly complexed by the exopolymer, but low concentrations of metal ions, such as those anticipated in the environment, will not change the conformational structure. At higher pHs and high concentrations of metals, such as those at a source term, the conformation of PGA is likely to be in a beta sheet structure. This data suggests that exopolymers will interact with actinides in the environment in a concentration dependent fashion that may lead to either enhanced mobilization or immobilization. 